\title{
Fourier Coefficients of Mathieu Functions in Stable Regions*
}

\author{
Henning Früchting**
}

(October 25, 1968)

\begin{abstract}
A method for calculating the Fourier coefficients of Mathieu functions in stable regions based on the method of bisection and Miller's recurrence algorithm is introduced. Some Fourier coefficients are calculated and compared with those given earlier by Tamir and Wang. It is shown that the method of Tamir and Wang fails for indices in the neighborhood of integers.
\end{abstract}

Key Words: Bisection method; eigenvalues; Fourier coefficients; Mathieu functions.

It is known from the theory of Mathieu functions [1] ${ }^{1}$ that the Floquet solution of the differential equation

$$
y^{\prime \prime}(z)+\left(\lambda-2 h^{2} \cos 2 z\right) y(z)=0
$$

with characteristic exponent $\nu$ has the Fourier expansion

$$
m e_{\nu}\left(z, h^{2}\right)=\sum_{n=-\infty}^{\infty} c_{2 n}^{\nu}\left(h^{2}\right) e^{i(\nu+2 n) z}
$$

The Fourier coefficients satisfy the following three-term recurrence formula:

$$
-h^{2} c_{2 n+2}^{\nu}\left(h^{2}\right)+\left[\lambda-(\nu+2 n)^{2}\right] c_{2 n}^{\nu}\left(h^{2}\right)-h^{2} c_{2 n-2}^{\nu}\left(h^{2}\right)=0 .
$$

Continued fraction expansions for the eigenvalues $\lambda=\lambda\left(\nu, h^{2}\right)$ and for the Fourier coefficients $c_{2 n}^{\nu} / c_{0}^{\nu}$ are given by Tamir and $\mathrm{W}$ ang in [2]. These relations are valid both in the stable ( $\nu$ real) and unstable ( $\nu$ complex) regions. $F(\nu) \equiv c_{2 n}^{\nu} / c_{0}^{\nu}$ is numerically evaluated and depicted. It is seen from the graphs in [2] that the given formulas for $c_{2 n}^{\nu} / c_{0}^{\nu}$ are not particularly suitable for numerical evaluation in the stable regions. Representations for $F(\nu)=c_{2 n}^{\nu} / c_{0}^{\nu}$ derived from other numerical methods will now be introduced.

The eigenvalues $\lambda$ of Mathieu's differential equation can be determined as the eigenvalues of the symmetrical tridiagonal matrix of the homogeneous system of eq (3) with the help of the bisection method [3]. Then the Fourier coefficients are obtainable from the recurrence formula (3) under consideration of stability (Miller's recurrence algorithm [4,5]). The accuracy of this method depends principally upon the number of equations used.

Numerical work was carried out to single precision on the computer of the Technische Hochschule Darmstadt. For evaluating the eigenvalues $\lambda$, a modified version of the bisection method [6] was used. This modification prohibits overflow. Also, a numerical test ensures that the trunca-

\footnotetext{
${ }^{1}$ Figures in brackets indicate the literature references at the end of this paper.

*An invited paper.

**Present address: Lehrstuhl für Theoretische Elektrotechnik, Technische Hochschule, 61 Darmstadt, Schlossgartenstrasse 2, Germany.
} 
tion error is smaller than the rounding error of the bisection method, when the number of equations used is $\left[\nu+h^{2}\right]+8$. The equations are set up in such a manner that with increasing $n$ the equation with the least value of $|\nu+2 n|$ is in the middle.
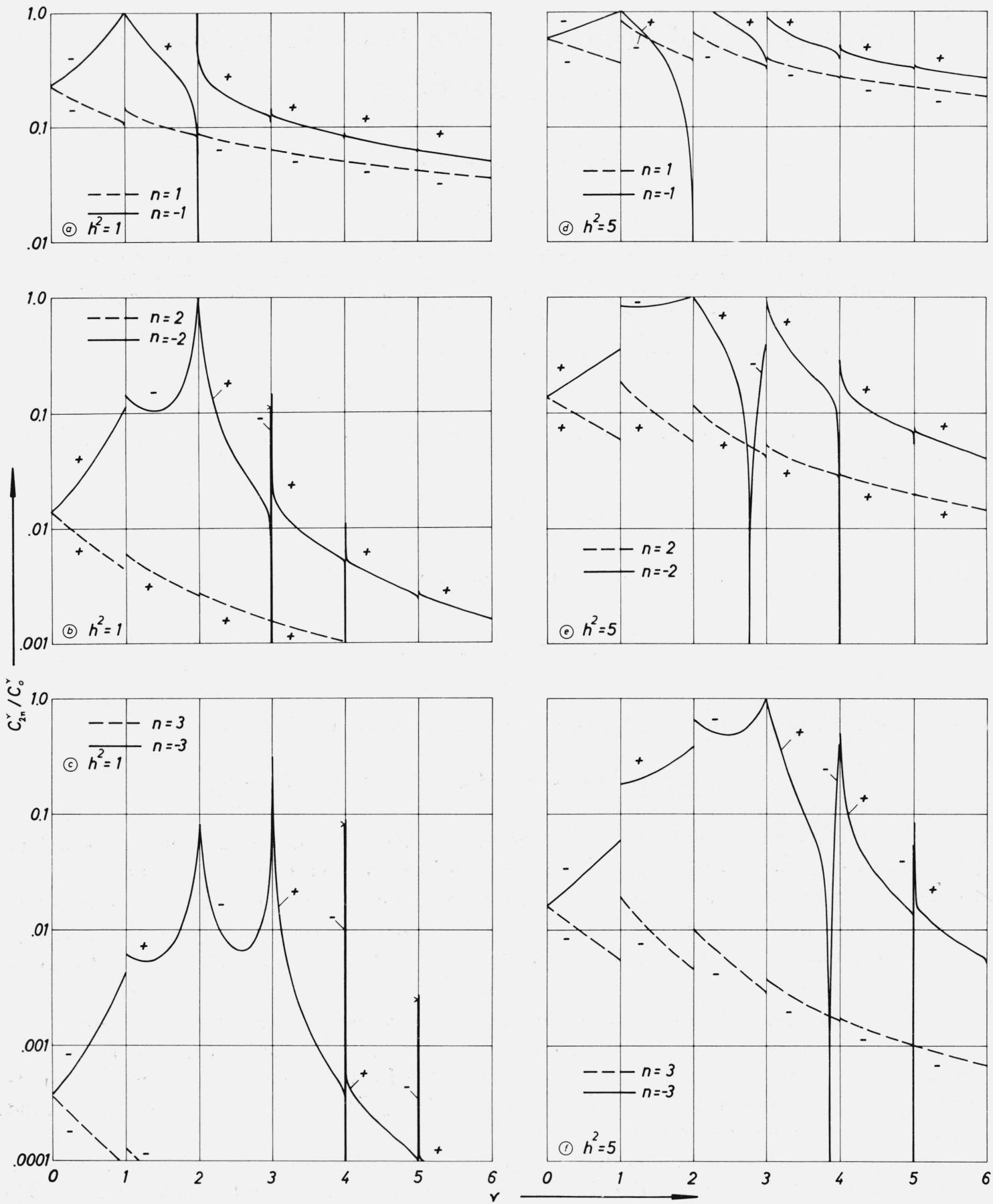

FigURE 1: Modulus and sign of $\mathrm{c}_{2 \mathrm{n}}^{\nu}\left(\mathrm{h}^{2}\right) / \mathrm{c}_{0}^{\nu}\left(\mathrm{h}^{2}\right)$ as a function of $\nu$ for two values of the parameter $\mathrm{h}^{2}$. 
To avoid overflow in applying (3), this relation is rewritten as a recurrence formula for the quotients $c_{2 n}^{\nu} / c_{2 n-2}^{\nu}$, given by

$$
\frac{c_{2 n+2}^{\nu}}{c_{2 n}^{\nu}}-\frac{\lambda-(\nu+2 n)^{2}}{h^{2}}+\left(\frac{c_{2 n}^{\nu}}{c_{2 n-2}^{\nu}}\right)^{-1}=0
$$

compare [7]. The starting values $c_{2 N}^{\nu} / c_{2 N-2}^{\nu}$ and $c_{-2 N}^{\nu} / c_{-2 N+2}^{\nu}$. were evaluated from continued fraction expansions obtained from (4) with $c_{2(N+m)}^{\nu}$ and $c_{-2(N+m)}^{\nu}$ set equal to zero. A numerical test determines the number of elements, $m$, to be used.

Figure 1 shows that as a function of $\nu, c_{2 n}^{\nu} / c_{0}^{\nu}$ is continuous for noninteger $\nu$. It has $n$ zeros; they lie near integer values of $\nu$ for small $h^{2}$ (fig. la-c). For increasing values of $h^{2}$ the zeros move toward smaller $\nu$ (fig. $1 \mathrm{~d}-\mathrm{f}$ ). For interger $\nu$, however, $c_{2 n}^{\nu} / c_{0}^{\nu}$ is discontinuous. The relation

$$
c_{r-m}^{m}\left(h^{2}\right)=c_{-r-m}^{m}\left(h^{2}\right)
$$

in which $r$ is an arbitrary integer, is valid for the limiting value from the right, giving $\lambda=a_{m}$, say, and $\nu=m, m=0,1, \ldots$.. Similarly,

$$
c_{r-m}^{m}\left(h^{2}\right)=-c_{-r-m}^{m}\left(h^{2}\right)
$$

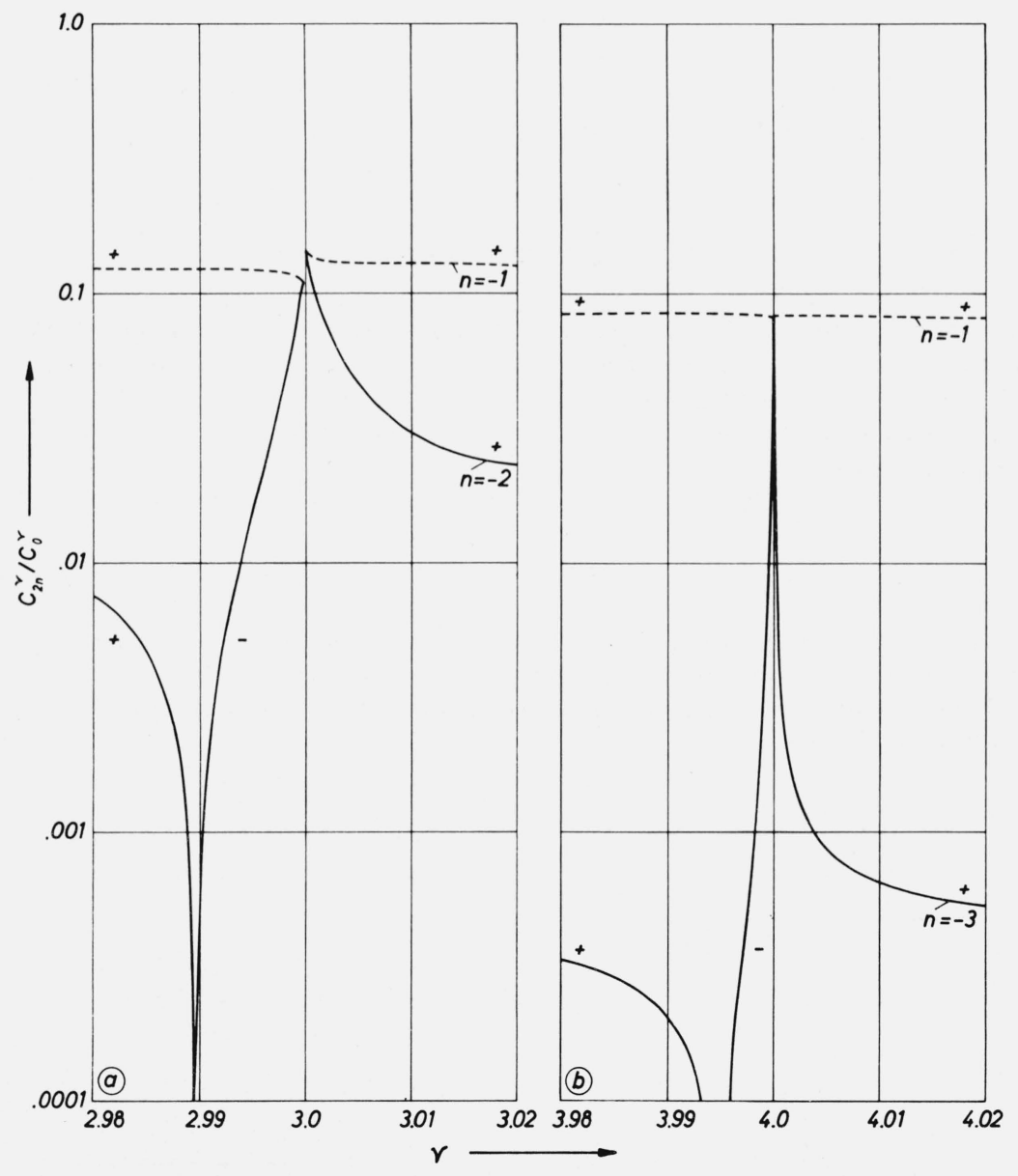

FIGURE 2: Modulus and sign of $\mathrm{c}_{2 \mathrm{n}}^{\nu}(1) / \mathrm{c}_{0}^{\nu}(1)$ in the region $\nu \approx \mathrm{m}$.

$\begin{array}{ll}\text { a. } m=3 \text {, illustrating } c_{-2}^{\nu} / c_{0}^{\nu} \text { and } c_{-4}^{\nu} / c_{0}^{\nu} & \text { b. } m=4 \text {, illustrating } c_{-2}^{\nu} / c_{0}^{\nu} \text { and } c_{-6}^{\nu} / c_{0}^{\nu}\end{array}$ 
is valid for the limiting value from the left, giving $\lambda=b_{m}$, say, and $\nu=m, m=1,2, \ldots$.; compare [1], p. 116, and [2]. In particular, for even values of $m$ we have from (6)

$$
c_{-m}^{m}\left(h^{2}\right)=-c_{-m}^{m}\left(h^{2}\right)=0 .
$$

Fourier coefficients with other indices coinciding according to eqs (5) and (6) with $\nu \rightarrow m$ are shown in figure 2. The absolute values of the Fourier coefficients with negative indices vary rapidly in the region $m-\epsilon<\nu<m, \epsilon \ll 1$.

Tamir and Wang [2] calculated only the zeros of $c_{2 n}^{\nu} / c_{0}^{\nu}$ which result from eq (6a), although by use of (5) and (6) it is also seen that for $\nu \neq m c_{2 n}^{\nu} / c_{0}^{\nu}$ can vanish. Thus the graphs in [2] become false. This leads to an erroneous calculation of Mathieu functions with indices in the neighborhood of integers.

\section{Summary}

A method for calculating the Fourier coefficients of Mathieu functions in stable regions based on the method of bisection and Miller's recurrence algorithm is introduced. Some Fourier coefficients are calculated and compared with those given earlier by Tamir and Wang. It is shown that the method of Tamir and Wang fails for indices in the neighborhood of integers.

The author is grateful to Dr.-Ing. G. Piefke for suggesting this work, which was sponsored by the Deutsche Forschungsgemeinschaft.

\section{References}

[1] Meixner, J., and Schäfke, F. W., Mathieusche Funktionen und Sphäroidfunktionen (Springer-Verlag, Berlin, 1954).

[2] Tamir, T., and Wang, H. C., Characteristic relations for nonperiodic solutions of Mathieu's equation, J. Res. NBS 69B (Math. and Math. Phys.) Nos. 1 and 2 (1965).

[3] Wilkinson, J. H., Calculation of the eigenvalues of a symmetric tridiagonal matrix by the method of bisection, Num. Mathematik 4, 362-367 (1962).

[4] Abramowitz, M., and Stegun, I. A., Generation of Bessel functions on high speed computers, M.T.A.C. 11, 255-257 (1957).

[5] Gautschi, W., Instability of linear second-order difference equations, Information Processing, Proc. IFIP Congress Munich, 207 (1963).

[6] Barth, W., Martin, R. S., and Wilkinson, J. H., Handbook series linear algebra. Calculation of the eigenvalues of a symmetric tridiagonal matrix by the method of bisection, Num. Mathematik 9,386-393 (1966-1967).

[7] Corbato, F. J., On the computation of auxiliary functions for two-center integrals by means of a high-speed computer, J. Chem. Phys. 24, 452-453 (1956).

(Paper 73B1-282) 